Pacific Journal of Mathematics

SPECTRAL SYNTHESIS IN HYPERGROUPS

THE 


\title{
SPECTRAL SYNTHESIS IN HYPERGROUPS
}

\author{
Ajit Kaur Chilana and Kenneth A. Ross
}

A commutative hypergroup $K$ is, roughly speaking, a space in which the product of two elements is a probability measure. Such spaces have been studied by Dunkl, Jewett, and Spector. Examples include locally compact abelian groups and double-coset spaces. $K$ has a Haar measure $m$ (Spector). It is shown that for several important classes of hypergroups the structure space of $L^{1}(m)$ is a hypergroup $\hat{K}$. For such spaces, $L^{1}(m)$ is shown to be regular, in fact, superregular, and to have good approximate units. A WienerTauberian theorem is given. Points in the center of $\hat{K}$ are shown to be strong Ditkin sets. Examples (due essentially to Reiter and Naimark) show that not all points in $\hat{K}$ need be spectral sets.

1. Introduction. The purpose of this paper is to determine to what extent results for the group algebra of a locally compact abelian group carry over to commutative hypergroups. The theory of topological hypergroups was initiated by Dunkl [3], Jewett [6], and Spector [12] and has recently received a good deal of attention from harmonic analysts. Throughout the paper, $K$ will denote a commutative locally compact hypergroup such that $K^{\wedge}$ is a hypergroup under pointwise operations. Being commutative, $K$ admits a Haar measure $m$, as shown by Spector [13]. The convolution algebra $L^{1}(m)=L^{1}(K)$ can be identified with the pointwise algebra $A\left(K^{\wedge}\right)$ of Fourier transforms on $K^{\wedge}$. The main reference will be Jewett [6] who calls hypergroups "convos." A survey of the subject appears in [10].

In $\S 2$ we establish some basic facts about $A\left(K^{\wedge}\right) . A\left(K^{\wedge}\right)$ is shown to be a regular algebra of functions on $K^{\wedge}$; in fact, $A\left(K^{\wedge}\right)$ is super-regular (2.9). It is shown that $A\left(K^{\wedge}\right)$ has some useful approximate units. A Wiener-Tauberian theorem is given. Some results on spectral synthesis are given in $\S 3$. The main result asserts that points in the center of $K^{\wedge}$ are strong Ditkin sets. Several examples are discussed in $\S 4$. In particular, it is observed that, in general, points of $K^{\wedge}$ need not be spectral sets. It is also observed that there exists nondiscrete $K^{\wedge}$ such that every closed subset is a Calderón set.

1.1. As remarked above, we assume throughout that

$\left(H_{1}\right) K^{\wedge}$ is a hypergroup under pointwise multiplication.

In (3.5)-(3.13) we impose another hypothesis which we now discuss. 
The hypergroup $K^{\wedge}$ need not be the structure space of $A\left(K^{\wedge}\right)$. In fact, the structure space for $A\left(K^{\wedge}\right) \approx L^{1}(K)$ is the space $\mathscr{Z}_{b}(K)$ of all bounded continuous multiplicative functions on $K[6,6.3]$. We always have $K^{\wedge} \subseteq \mathscr{Z}_{b}(K)$, but in (3.5)-(3.13) we assume that

$\left(H_{2}\right) \quad K^{\wedge}=\mathscr{X}_{b}(K)$.

We devote the remainder of $\S 1$ to showing that $\left(H_{1}\right)$ and $\left(H_{2}\right)$ hold for several important classes of hypergroups.

1.2. To start with, observe that $\left(H_{1}\right)$ and $\left(H_{2}\right)$ hold if $K$ is a locally compact abelian group. Now let $G$ be a locally compact abelian group and let $B$ be a subgroup of the automorphism group of $G$ having compact closure. Then the space $G_{B}$ of $B^{-}$-orbits is a hypergroup. Property $\left(H_{1}\right)$ holds for $G_{B}$ because $\left(G_{B}\right)^{\wedge}$ is isomorphic with $\left(G^{\wedge}\right)_{B}$ where $G^{\wedge}$ denotes the character group of $G$. Property $\left(H_{2}\right)$ also holds for $G_{B}$. See [11] for a more detailed discussion.

1.3. Compact hypergroups always satisfy $\left(H_{2}\right)[3,3.5]$ but $\left(H_{1}\right)$ can even fail for three-element hypergroups [3, 3.8], [6, 9.1C].

Proposition 1.4. Let $G$ be a Z-group, i.e. a group such that $G / Z$ is compact where $Z$ denotes the center of $G$. The hypergroup $K$ of conjugacy classes of $G$ satisfies $\left(H_{1}\right)$ and $\left(H_{2}\right)$. For each $x \in K$ let $x^{\sim}$ be defined on $K^{\wedge}$ by $x^{\sim}(\psi)=\overline{\psi(x)}$. Then $\mathfrak{X}_{b}\left(K^{\wedge}\right)=K^{\wedge}=$ $\left\{x^{\sim}: x \in K\right\}$. In particular, $K^{\wedge \wedge}$ is isomorphic with $K$ and $\left(H_{1}\right)$ and $\left(H_{2}\right)$ hold for $K^{\wedge}$.

Proof. As observed in [11], $K^{\wedge}$ can be identified with the space $\mathfrak{X}$ of normalized characters on $G$. It is shown that $\mathfrak{X}$ is a hypergroup in $[11,5.5]$ and so $K$ satisfies $\left(H_{1}\right)$. A theorem of Hulanicki (see $[16,4.12])$ shows that $K$ also satisfies $\left(H_{2}\right)$.

As noted in [6, 12.4], $x \rightarrow x^{\sim}$ is a homeomorphism of $K$ onto a closed subset of $K^{\wedge \wedge}$. Now consider $\chi \in \mathfrak{X}_{b}\left(K^{\wedge}\right)$. The fact that $\chi=x^{\sim}$ for some $x \in K$ follows directly from Theorem 2 in Kaniuth and Steiner [15]. To translate into their notation, observe that $E(G)=\mathfrak{X} \cong K^{\wedge}$ and that $\Re(G)=K$. For $\xi \in L^{1}\left(K^{\wedge}\right)$, let $h(\xi)=$ $\int_{K^{\wedge}} \xi(\psi) \chi(\bar{\psi}) d \psi$. Then $h$ is a nonzero multiplicative linear functional on $L^{1}\left(K^{\wedge}\right)$ and the theorem of Kaniuth and Steiner shows that $h(\xi)=\int_{K^{\wedge}} \xi(\psi) \psi(x) d \psi$ for some $x \in K$ and all $\xi \in L^{1}\left(K^{\wedge}\right)$. Then $\chi(\psi)=$ $\tilde{\psi}(x)=x^{\sim}(\psi)$ for $\psi \in K^{\wedge}$.

An interesting hypergroup for which both $\left(H_{1}\right)$ and $\left(H_{2}\right)$ fail is discussed in (4.8.) 
2. The algebra $A\left(K^{\wedge}\right)$. We begin by setting down some results that are straightforward consequences of basic results in [6]. As stated in $\S 1$, we assume throughout that $K$ is a commutative hypergroup and that $K^{\wedge}$ is a hypergroup under pointwise operations. The Plancherel measure on $K^{\wedge}$ will be denoted by $\pi$. Since $K^{\wedge}$ is a hypergroup, $\pi$ is the Haar measure for $K^{\wedge}$; see $[6,7.3,12.4]$. In view of $[6,7.3 I]$ the Fourier transform on $L^{1}(m) \cap L^{2}(m)$ extends to an isometry of $L^{2}(m)$ onto $L^{2}(\pi)$.

Lemma 2.1. If $f$ and $g$ are in $L^{2}(m)$, then $f^{\wedge}$ and $g^{\wedge}$ are in $L^{2}(\pi)$ and $f^{\wedge} * g^{\wedge}=(f g)^{\wedge}$. Moreover, $(\varphi, \psi) \rightarrow \phi * \psi$ is a jointly continuous mapping from $L^{2}(\pi) \times L^{2}(\pi)$ onto $A\left(K^{\wedge}\right)$.

LEMMA 2.2. If $f$ belongs to $L^{1}(m)$ or $L^{2}(m)$ and if $\gamma \in K^{\wedge}$, then $(f \bar{\gamma})^{\wedge}=\left(f^{\wedge}\right)_{r}$. If $f$ belongs to $L^{1}(m)$ and $y \in K$, then $\left(f_{y}\right)^{\wedge}(\gamma)=\gamma(y) f^{\wedge}(\gamma)$ for all $\gamma \in K^{\wedge}$.

The next lemma follows from (2.2B) and $(5.4 \mathrm{H})$ in [6].

Lemma 2.3. For $f$ in $L^{1}(m)$, the mapping $y \rightarrow f_{y}$ is continuous from $K$ into $L^{1}(m)$.

Lemma 2.4. If $f, g$ are in $L^{1}(m)$ and $y \in K$, then

$$
(f * g)_{y}=f *\left(g_{y}\right)=\left(f_{y}\right) * g .
$$

The regularity of $A\left(K^{\wedge}\right)$ is a consequence of the next lemma, whose statement and proof will be familiar.

LEMMA 2.5. Let $E$ be a compact subset of $K^{\wedge}$ and let $V$ be a symmetric set such that $\pi(V)>0$ and such that its closure $V^{-}$is compact. Then there is a function $\varphi$ in $A\left(K^{\wedge}\right)$ such that $0 \leqq \varphi \leqq 1$, $\varphi(\gamma)=1$ for $\gamma \in E$ and $\varphi(\gamma)=0$ for $\gamma \notin E * V * V$. Also, we have $\|\varphi\|_{A}^{2} \leqq \pi(E * V) / \pi(V)$.

Proof. Let $\xi_{V}$ and $\xi_{E_{*} V}$ denote the characteristic functions of $V$ and $E * V$, respectively, and let $\varphi=\pi(V)^{-1} \xi_{V} * \xi_{E_{*} V}$. Lemma 2.1 shows that $\varphi$ belongs to $A\left(K^{\wedge}\right)$. For $\gamma \in K^{\wedge}$, we have

$$
\varphi(\gamma)=\pi(V)^{-1} \int_{V}\left(p_{\gamma} * p_{\gamma^{\prime}}\right)(E * V) d \pi\left(\gamma^{\prime}\right) ;
$$

$p_{\gamma}$ denotes the point mass at $\gamma$. Since each $p_{\gamma} * p_{\gamma^{\prime}}$ is a probability measure, we see that $0 \leqq \varphi \leqq 1$. If $\gamma \in E$, then $\operatorname{supp}\left(p_{r} * p_{\gamma^{\prime}}\right) \leqq E * V$ for all $\gamma^{\prime} \in V$, from which it follows that $\varphi(\gamma)=1$. If $\varphi(\gamma) \neq 0$, then $\left(p_{r} * p_{r^{\prime}}\right)(E * V)>0$ for some $\gamma^{\prime} \in V$. From (4.1B) in [6] it follows that 
$\gamma \in \bar{\gamma}^{\prime} * E * V \subseteq E * V * V$. The norm inequality is easily verified.

The subscript " 00 " on a family of functions restricts the family to its members having compact support.

THEOREM 2.6. $A\left(K^{\wedge}\right)$ is a regular Banach algebra of functions on $K^{\wedge}$. Moreover, $A_{00}\left(K^{\wedge}\right)$ is dense in $A\left(K^{\wedge}\right)$.

Proof. Lemma 2.5 establishes the regularity. Lemma 2.1 implies that $C_{00}\left(K^{\wedge}\right) * C_{c 0}\left(K^{\wedge}\right)$ is dense in $A\left(K^{\wedge}\right)$ and $[6,3.2 \mathrm{~B}]$ implies that $C_{00}\left(K^{\wedge}\right) * C_{00}\left(K^{\wedge}\right) \subseteq A_{00}\left(K^{\wedge}\right)$.

We now obtain two useful approximate units for $L^{1}(m)$, i.e., for $A\left(K^{\wedge}\right)$. We use the notation " " to signify involution.

LEMMA 2.7. Let $f$ be in $L^{1}(m)$ and $\varepsilon>0$. There is a neighborhood $V$ of the identity $e$ in $K$ such that for every nonnegative Borel function $u$ supported by $V$ and satisfying $\int_{K} u(\check{x}) d m(x)=1$, we have $\|f-f * u\|_{1}<\varepsilon$.

Proof. By Lemma 2.3 there is a symmetric neighborhood $V$ of $e$ such that $\left\|f_{y}-f\right\|_{1}<\varepsilon$ for $y \in V$. If $u$ is as indicated, then

$$
f * u(x)-f(x)=\int_{K}\left[f_{y}(x)-f(x)\right] u(\check{y}) d m(y)
$$

for $x \in K$, and therefore

$$
\begin{aligned}
\|f * u-f\|_{1} & \leqq \int_{K} \int_{K}\left|f_{y}(x)-f(x)\right| u(\check{y}) d m(y) d m(x) \\
& =\int_{K}\left\|f_{y}-f\right\|_{1} u(\check{y}) d m(y)<\varepsilon .
\end{aligned}
$$

THEOREM 2.8. $A\left(K^{\wedge}\right)$ has an approximate unit $\left\{\varphi_{\alpha}\right\}$ such that each $\varphi_{\alpha}$ belongs to $A_{00}\left(K^{\wedge}\right)$ and $\left\|\varphi_{\alpha}\right\|_{A}=1$ for all $\alpha$. If $K$ is metrizable, $\left\{\varphi_{\alpha}\right\}$ can be chosen as a sequence.

Proof. Let $\mathscr{C}$ be a basis of neighborhoods at $e$ consisting of compact symmetric sets. We direct the net by $D=\{(U, \delta): U \in \mathscr{U}$, $0<\delta<1\}$ where $\left(U_{1}, \delta_{1}\right) \geqq\left(U_{2}, \delta_{2}\right)$ signifies $U_{1} \leqq U_{2}$ and $\delta_{1} \leqq \delta_{2}$. For $U \in \mathscr{U}$, let $f_{U}=m(U)^{-1} \xi_{U}$. For $\alpha=(U, \delta)$, use Theorem 2.6 to select $\psi_{\alpha}$ in $A_{00}\left(K^{\wedge}\right)$ such that $\left\|\hat{f}_{U}-\psi_{\alpha}\right\|_{A}<\delta$. Finally, define $\varphi_{\alpha}=\left\|\psi_{\alpha}\right\|_{A}^{-1} \psi_{\alpha}$. Some routine estimates and an application of Lemma 2.7 show that $\left\{\varphi_{\alpha}\right\}$ is an approximate unit for $A\left(K^{\wedge}\right)$.

Before obtaining our second approximate unit, we use Theorem 2.8 to show that $A\left(K^{\wedge}\right)$ is super-regular. 
THEOREM 2.9. Let $E$ be a compact subset of $K^{\wedge}$ and let $\varepsilon>0$. Then there is a function $\psi$ in $A_{00}\left(K^{\wedge}\right)$ such that $\psi(\gamma)=1$ for $\gamma \in E$ and $\|\psi\|_{A}<1+\varepsilon$.

Proof. By Lemma 2.5 there exists $\varphi$ in $A_{00}\left(K^{\wedge}\right)$ such that $\varphi=1$ on $E$. By (2.8) there exists $\varphi_{0}$ in $A_{00}\left(K^{\wedge}\right)$ such that $\left\|\varphi_{0}\right\|_{A}=1$ and $\left\|\varphi-\varphi \varphi_{0}\right\|_{A}<\varepsilon$. Now let $\psi=\varphi+\varphi_{0}-\varphi \varphi_{0}$.

THEOREM 2.10. $A\left(K^{\wedge}\right)$ has an approximate unit $\left\{\varphi_{\alpha}\right\}$ with the following properties. If $\varphi_{\alpha}=\hat{f}_{\alpha}$ for $f_{\alpha} \in L^{1}(m)$, then each $f_{\alpha}$ belongs to $C_{00}^{+}(K)$, each $\varphi_{\alpha}$ belongs to $L^{1}(\pi), \varphi_{\alpha} \geqq 0$, and $\left\|\varphi_{\alpha}\right\|_{A}=1$ for all $\alpha$. If $K$ is metrizable, $\left\{\varphi_{\alpha}\right\}$ can be chosen as a sequence.

Proof. Our directed set will be a basis $\mathscr{U}$ of compact symmetric neighborhoods of $e$. For each $U$ in $\mathscr{U}$, select $V$ in $\mathscr{C}$ satisfying $V * V \leqq U$. Let $g_{V}=m(V)^{-1} \xi_{V}, f_{U}=g_{V} * g_{V}$, and $\varphi_{U}=\hat{f}_{U}$. Then $f_{U}$ is easily seen to be in $C_{00}^{+}(K)$. Since $\hat{g}_{V}$ belongs to $L^{2}(\pi), \varphi_{U}=\left(\hat{g}_{V}\right)^{2}$ belongs to $L^{1}(\pi)$. Since $V$ is symmetric and $g_{V}$ is real-valued, $\hat{g}_{V}$ is real-valued and so $\varphi_{U} \geqq 0$. Since $\hat{g}_{V}(1)=1$, we have

$$
1=\varphi_{U}(1) \leqq\left\|\varphi_{U}\right\|_{u} \leqq\left\|\varphi_{U}\right\|_{A}=\left\|f_{U}\right\|_{1} \leqq\left\|g_{V}\right\|_{1}\left\|g_{V}\right\|_{1}=1,
$$

i.e., $\left\|\varphi_{U}\right\|_{A}=1$. Finally, Lemma 2.7 implies that $\left\{\varphi_{U}\right\}$ is an approximate unit for $A\left(K^{\wedge}\right)$.

THEOREM 2.11. A subset I of $L^{1}(K)$ is a closed ideal if and only if it is a closed translation-invariant subspace.

Proof. Let $I$ be a closed ideal, $f \in I, y \in K$. If $\left\{g_{\alpha}\right\}$ is an approximate unit for $L^{1}(K)$, then $f_{y}=\lim _{\alpha}\left(f_{y}\right) * g_{\alpha}$. But by (2.4), each $\left(f_{y}\right) * g_{\alpha}=f *\left(g_{\alpha}\right)_{y}$ is in $I$ and so $f_{y} \in I$.

Now suppose that $I$ is a closed translation-invariant subspace and let $f \in I, g \in L^{1}(K), \varepsilon>0$. Select $h \in C_{00}(K)$ so that $\|f\|_{1}\|g-h\|_{1}<$ $\varepsilon / 2$. Use Lemma 2.3 to partition the compact set $\operatorname{supp}(h) \cup \operatorname{Supp}(h)^{\smile}$ into Borel sets $\left\{B_{j}\right\}_{j=1}^{n}$ so that

$$
x, y \in B_{j} \quad \text { imply } \quad\left\|h^{\smile}\right\|_{1}\left\|f_{y}-f_{x}\right\|_{1}<\varepsilon / 2 .
$$

Select $x_{j} \in B_{j}$ and set $c_{j}=\int_{B_{j}} h(\check{x}) d m(x)$. For $x \in K$, we have

$$
(h * f)(x)-\sum_{j=1}^{n} c_{j}\left(f_{x_{j}}\right)(x)=\sum_{j=1}^{n} \int_{B_{j}}\left[f_{y}(x)-f_{x_{j}}(x)\right] h(\check{y}) d m(y),
$$

from which it follows that $\left\|h * f-\sum_{j=1}^{n} c_{j}\left(f_{x_{j}}\right)\right\|_{1}<\varepsilon / 2$. Hence $\left\|g * f-\sum_{j=1}^{n} c_{j}\left(f_{x_{j}}\right)\right\|_{1}<\varepsilon$. Thus $g * f \in I$ since $I$ is translation-invariant and closed. 
Here is a Wiener-Tauberian theorem for hypergroups.

THEOREM 2.12. If $f$ belongs to $L^{1}(K)$ and if $\hat{f}$ vanishes nowhere on $K^{\wedge}$, then the closed translation-invariant subspace of $L^{1}(K)$ generated by $f$ is $L^{1}(K)$ itself.

We omit the direct proof which is similar to that for the group case. If $K^{\wedge}$ is the structure space $\mathscr{Z}_{b}(K)$ for $L^{1}(K)$, then this theorem follows from the abstract Tauberian theorem [5, 39.27] in view of Theorem 2.6. See the discussion in (1.1).

3. Synthesis in $A\left(K^{\wedge}\right)$. As before, $K$ denotes a commutative hypergroup such that $K^{\wedge}$ is also a hypergroup. The center $Z(K)$ of $K$ consists of all $x$ in $K$ such that $\operatorname{supp}\left(p_{x} * p_{y}\right)$ is a singleton for each $y \in K$. The center $Z\left(K^{\wedge}\right)$ of $K^{\wedge}$ is the set of $\psi$ in $K^{\wedge}$ such that $|\psi|=1$. A detailed study of centers appears in [11]. The main result in this section, and in this paper, asserts that each point in $Z\left(K^{\wedge}\right)$ is a strong Ditkin set for the algebra $A\left(K^{\wedge}\right)$; see (3.3) and (3.8). We begin with two lemmas.

LEMMA 3.1. Let $F$ be a compact symmetric subset of $K$ and $\varepsilon>0$. If $H \subseteq\left\{\gamma \in K^{\wedge}:|\gamma(y)-1|<\varepsilon\right.$ for all $\left.y \in F\right\}, \pi(H)<\infty$, and if $\hat{g}=\xi_{H}$, then $\left\|g_{x}-g\right\|_{2} \leqq \varepsilon \pi(H)^{1 / 2}$ for $x \in F$.

Proof. By Lemma 2.2, we have

$$
\begin{aligned}
\left\|g_{x}-g\right\|_{2}^{2} & =\left\|\hat{g}_{x}-\hat{g}\right\|_{2}^{2}=\int_{K^{\wedge}}|\gamma(x) \hat{g}(\gamma)-\hat{g}(\gamma)|^{2} d \pi(\gamma) \\
& =\int_{H}|\gamma(x)-1|^{2} d \pi(\gamma) \leqq \varepsilon^{2} \pi(H) .
\end{aligned}
$$

LEMMA 3.2. If $F$ is a compact symmetric subset of $K$ and $\delta>0$, then there exists $g \in L^{1}(K)$ such that

(i) $\|g\|_{1}<2$,

(ii) $\hat{g}=1$ on a neighborhood of 1 ,

(iii) $\int_{K}|g(y * \check{x})-g(y)| d m(y) \leqq \delta$ for $x \in F$.

Proof. Let $\varepsilon=\delta / 12$ and let

$$
\Phi=\left\{\gamma \in K^{\wedge}:|\gamma(y)-1|<\varepsilon \quad \text { for } \quad y \in F\right\} .
$$

Since $K^{\wedge}$ has the compact-open topology, $\Phi$ is a neighborhood of 1 and so $\Phi$ contains an open symmetric neighborhood $H_{1}$ of 1 having compact closure. Since $\pi$ is a regular measure on $K^{\wedge}$, there exists a compact symmetric neighborhood $H_{2}$ of 1 such that 
$H_{2} \subseteq H_{1}$ and $\pi\left(H_{2}\right)>(1 / 4) \pi\left(H_{1}\right)$. By $[6,3.2 \mathrm{D}]$, there is a neighborhood $\Psi$ of 1 such that $\Psi * H_{2} \subseteq H_{1}$. Now there exist $g_{1}, g_{2}$ in $L^{2}(K)$ such that $\hat{g}_{i}=\xi_{H_{i}}, i=1,2$. Finally we put $g=\pi\left(H_{2}\right)^{-1} g_{1} g_{2}$. Observe that $\left\|g_{i}\right\|_{2}^{2}=\pi\left(H_{i}\right)$ and so

$$
\|g\|_{1}=\pi\left(H_{2}\right)^{-1}\left\|g_{1} g_{2}\right\|_{1} \leqq \pi\left(H_{2}\right)^{-1}\left\|g_{1}\right\|_{2}\left\|g_{2}\right\|_{2}=\left[\pi\left(H_{1}\right) / \pi\left(H_{2}\right)\right]^{1 / 2}<2 .
$$

To check (ii), consider $\psi \in \Psi$. Then

$$
\begin{aligned}
\pi\left(H_{2}\right) \hat{g}(\psi) & =\left(g_{1} g_{2}\right)^{\wedge}(\psi)=\hat{g}_{1} * \hat{g}_{2}(\psi)=\int_{K^{\wedge}} \xi_{H_{1}}(\psi * \gamma) \xi_{H_{2}}(\bar{\gamma}) d \pi(\gamma) \\
& =\int_{H_{2}} \xi_{H_{1}}(\psi * \gamma) d \pi(\gamma)=\int_{H_{2}} \int_{K^{\wedge}} \xi_{H_{1}} d\left(p_{\psi} * p_{\gamma}\right) d \pi(\gamma)
\end{aligned}
$$

For $\gamma \in H_{2}$, we have $\operatorname{supp}\left(p_{\psi} * p_{\gamma}\right) \subseteq \Psi * H_{2} \subseteq H_{1}$ and so $\xi_{H_{1}}$ is identically 1 on $\operatorname{supp}\left(p_{\psi} * p_{\gamma}\right)$. Therefore $\pi\left(H_{2}\right) \hat{g}(\psi)=\pi\left(H_{2}\right)$, i.e., $\hat{g}(\psi)=1$. Thus $\hat{g}=1$ on $\Psi$ and (ii) holds.

Since $F$ is symmetric, it suffices to prove (iii) for $x$ in place of $\check{x}$. Observe that

$$
\begin{aligned}
\pi\left(H_{2}\right) & {[g(y * x)-g(y)]=\left(g_{1} g_{2}\right)(y * x)-g_{1}(y) g_{2}(y) } \\
= & \int_{K}\left[g_{1}(z)-g_{1}(y)\right]\left[g_{2}(z)-g_{2}(y)\right] d\left(p_{y} * p_{x}\right)(z) \\
& \quad+g_{1}(y)\left[\left(g_{2}\right)_{x}(y)-g_{2}(y)\right]+g_{2}(y)\left[\left(g_{1}\right)_{x}(y)-g_{1}(y)\right] \\
\equiv & G_{1}(y)+G_{2}(y)+G_{3}(y) .
\end{aligned}
$$

Using Lemma 3.1, we obtain

$$
\begin{aligned}
\int_{K}\left|G_{2}\right| d m & =\left\|g_{1}\left[\left(g_{2}\right)_{x}-g_{2}\right]\right\|_{1} \\
& \leqq\left\|g_{1}\right\|_{2}\left\|\left(g_{2}\right)_{x}-g_{2}\right\|_{2} \leqq \pi\left(H_{1}\right)^{1 / 2} \varepsilon \pi\left(H_{2}\right)^{1 / 2}
\end{aligned}
$$

Similarly, we have

$$
\int_{K}\left|G_{3}\right| d m \leqq \varepsilon \pi\left(H_{1}\right)^{1 / 2} \pi\left(H_{2}\right)^{1 / 2} .
$$

Estimation of the integral of $G_{1}$ is more delicate. By Hölder's inequality, we have $\left|G_{1}(y)\right|^{2} \leqq A_{1}(y) A_{2}(y)$ where

$$
A_{i}(y)=\int_{K}\left|g_{i}(z)-g_{i}(y)\right|^{2} d\left(p_{y} * p_{x}\right)(z) .
$$

Now

$$
\begin{aligned}
A_{i}(y) & =\int_{K}\left[\left|g_{i}(z)\right|^{2}-2 \operatorname{Re} g_{i}(z) \overline{g_{i}(y)}+\left|g_{i}(y)\right|^{2}\right] d\left(p_{y} * p_{x}\right)(z) \\
& =\left(\left|g_{i}\right|^{2}\right)_{x}(y)-2 \operatorname{Re} \overline{g_{i}(y)}\left(g_{i}\right)_{x}(y)+\left|g_{i}(y)\right|^{2} \\
& =\left(\left|g_{i}\right|^{2}\right)_{x}(y)-\left|\left(g_{i}\right)_{x}(y)\right|^{2}+\left|\left(g_{i}\right)_{x}(y)-g_{i}(y)\right|^{2} .
\end{aligned}
$$


By $[6,3.3 B]$, we have

$$
\int_{K}\left(\left|g_{i}\right|^{2}\right)_{x} d m \leqq \int_{K}\left|g_{i}\right|^{2} d m=\left\|g_{i}\right\|_{2}^{2}
$$

and so

$$
\begin{gathered}
\int_{K}\left[\left(\left|g_{i}\right|^{2}\right)_{x}-\left|\left(g_{i}\right)_{x}\right|^{2}\right] d m \leqq\left\|g_{i}\right\|_{2}^{2}-\left\|\left(g_{i}\right)_{x}\right\|_{2}^{2} \\
=\left[\left\|g_{i}\right\|_{2}+\left\|\left(g_{i}\right)_{x}\right\|_{2}\right]\left[\left\|g_{i}\right\|_{2}-\left\|\left(g_{i}\right)_{x}\right\|_{2}\right] \\
\quad \leqq 2\left\|g_{i}\right\|_{2}\left\|g_{i}-\left(g_{i}\right)_{x}\right\|_{2} .
\end{gathered}
$$

Also

$$
\int_{K}\left|\left(g_{i}\right)_{x}-g_{i}\right|^{2} d m=\left\|\left(g_{i}\right)_{x}-g_{i}\right\|_{2}^{2} \leqq 2\left\|g_{i}\right\|_{2}\left\|\left(g_{i}\right)_{x}-g_{i}\right\|_{2}
$$

and so

$$
\int_{K} A_{i} d m \leqq 4\left\|g_{i}\right\|_{2}\left\|\left(g_{i}\right)_{x}-g_{i}\right\|_{2} \leqq 4 \pi\left(H_{i}\right)^{1 / 2} \varepsilon \pi\left(H_{i}\right)^{1 / 2} .
$$

By Hölder's inequality again, we obtain

$$
\begin{aligned}
\int_{K}\left|G_{1}\right| d m & \leqq \int_{K} A_{1}^{1 / 2} A_{2}^{1 / 2} d m \leqq\left[\int_{K} A_{1} d m\right]^{1 / 2}\left[\int_{K} A_{2} d m\right]^{1 / 2} \\
& \leqq\left[16 \varepsilon^{2} \pi\left(H_{1}\right) \pi\left(H_{2}\right)\right]^{1 / 2}=4 \varepsilon \pi\left(H_{1}\right)^{1 / 2} \pi\left(H_{2}\right)^{1 / 2}
\end{aligned}
$$

Combining (1)-(4), we find

$$
\pi\left(H_{2}\right) \int_{K}|g(y * x)-g(y)| d m(y) \leqq 6 \varepsilon \pi\left(H_{1}\right)^{1 / 2} \pi\left(H_{2}\right)^{1 / 2}
$$

or

$$
\int_{K}|g(y * x)-g(y)| d m(y) \leqq 6 \varepsilon\left[\pi\left(H_{1}\right) / \pi\left(H_{2}\right)\right]^{1 / 2}<12 \varepsilon=\delta .
$$

The next theorem shows that the identity 1 of $K^{\wedge}$ may be viewed as a strong Ditkin set for $A\left(K^{\wedge}\right)$ even if $K^{\wedge}$ is not the structure space for $A\left(K^{\wedge}\right)$; see (1.1).

THEOREM 3.3. There is a net $\left\{f_{\alpha}\right\}$ in $L^{1}(K)$ such that

(i ) $\left\|f_{\alpha}\right\|_{1}<3$ for all $\alpha$,

(ii) if $f \in L^{1}(K)$ and $\hat{f}(1)=0$, then $\lim _{\alpha}\left\|f-f * f_{\alpha}\right\|_{1}=0$,

(iii) each $\hat{f}_{\alpha}$ vanishes in a neighborhood of 1 in $K^{\wedge}$ and has compact support.

Proof. Let $\left\{u_{\beta}\right\}$ be an approximate unit for $L^{1}(K)$ such that $\left\|u_{\beta}\right\|_{1}=1$ and $\widehat{u}_{\beta} \in A_{n_{0}}\left(K^{\wedge}\right)$ for all $\beta$; see (2.8). The net $\left\{f_{\alpha}\right\}$ will be directed by the set 


$$
D=\{(F, \delta, \beta): F \leqq K \text { compact symmetric, } \delta>0\}
$$

where $(F, \delta, \beta) \geqq\left(F^{\prime \prime}, \delta^{\prime}, \beta^{\prime}\right)$ signifies $F \supseteqq F^{\prime \prime}, \delta \leqq \delta^{\prime}, \beta \geqq \beta^{\prime}$. Given $\alpha=(F, \delta, \beta)$, select $g_{F, \delta}$ as in Lemma 3.2 and then define $f_{\alpha}=u_{\beta}$ $u_{\beta} * g_{F, \tilde{o}}$.

Properties (i) and (iii) are easy to verify. To verify (ii), consider $f \in L^{1}(K)$ such that $\hat{f}(1)=0$, and let $\varepsilon>0$. Select $\beta_{0}$ so that $\beta \geqq \beta_{0}$ implies $\left\|f-f * u_{\beta}\right\|_{1}<\varepsilon / 2$; select compact symmetric $F_{0}$ in $K$ so that $\int_{K \backslash F_{0} .}|f| d m<\varepsilon / 16$; and select $\delta_{0}$ so that $4 \delta_{0}\|f\|_{1}<\varepsilon$. If $\alpha_{0}=\left(F_{0}, \delta_{0}, \beta_{0}\right)$,

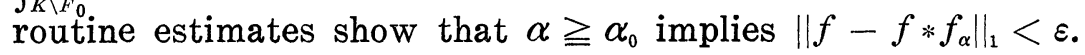

REMARK 3.4. The proof of Theorem 3.3 follows the same pattern as in the group case (see [5, 39.28], for example). The main new difficulty is that the relation $(f g)_{x}=f_{x} g_{x}$, familiar for functions on groups, does not in general hold for functions on hypergroups.

Concepts such as spectral set, strong Ditkin set and Calderón set are normally defined for subsets of the structure space of a regular commutative Banach algebra. For these reasons, we adopt

AN AdDitional Hypothesis 3.5. For the remainder of this section, we assume that $K^{\wedge}=\mathscr{P}_{b}(K)$. Thus $K$ will be a commutative hypergroup such that $K^{\wedge}$ is a hypergroup and $K^{\wedge}$ is the structure space of $L^{1}(K)$.

If $K$ is compact, then $K^{\wedge}$ is discrete and all its subsets are Calderón sets. So our results below on synthesis are of special interest only if $K$ is noncompact. In any case, $\{1\}$ is a strong Ditkin set for $L^{1}(K)$ by Theorem 3.3. Our next result and its corollary allow us to extend this result to points in $Z\left(K^{\wedge}\right)$. It should be compared with results of Rieffel [9, §4.2].

THEOREM 3.6. Let $h$ be a nonzero bounded continuous function on $K$, and define $J_{h}(g)=h g$ for each $g \in L^{1}(K)$. If $\gamma \in Z\left(K^{\wedge}\right)$, then $J_{r}$ is an isometric algebra isomorphism of $L^{1}(K)$ onto $L^{1}(K)$. Conversely, if $J_{h}$ preserves the convolution of $L^{1}(K)$, then $h$ belongs to $Z\left(K^{\wedge}\right)$.

Proof. The first statement is easily verified. For example, the identity $J_{\gamma}(f * g)=J_{\gamma}(f) * J_{\gamma}(g)$ is checked using the definition of convolution and the fact that $\gamma$ is constant with value $\gamma(x) \gamma(y)$ on $\operatorname{supp}\left(p_{x} * p_{y}\right)$ for each $x, y \in K$ and $\gamma \in Z\left(K^{\wedge}\right)[3,2.2]$.

For the converse, we first consider $x, y \in K$ and show that $h(s)=$ $h(x) h(y)$ for all $s \in \operatorname{supp}\left(p_{x} * p_{y}\right)$. Assume that $\beta=h(x) h(y)-h(s) \neq 0$ 
for some $s \in \operatorname{supp}\left(p_{x} * p_{y}\right)$ so that $[h(x) h(y)-h(s)] \bar{\beta}>0$. There exist compact neighborhoods $V$ and $W$ of $x$ and $y$, and $\eta>0$, so that

$$
\operatorname{Re}[h(v) h(w)-h(s)] \bar{\beta} \geqq \eta \quad \text { for } \quad v \in V, w \in W .
$$

Select a continuous nonnegative function $f$ so that $f(x)>0$ and $\operatorname{supp}(f) \subseteq V$, and let $g=\xi_{W}$. Since $x \in \operatorname{supp}\left(p_{s} * p_{y}^{v}\right)$ we have $f(s * \check{y})>0$. By the continuity of $w \mapsto f(s * \breve{w})$, we see that $f(s * \breve{w})>0$ in a neighborhood of $y$ and so

$$
\int_{W} f(s * \breve{w}) d m(w)>0 .
$$

Direct calculations show that

$$
(h f) *(h g)(s)=\int_{W} \int_{V} h(v) f(v) d\left(p_{s} * p_{w}^{v}\right)(v) h(w) d m(w)
$$

and

$$
h(f * g)(s)=h(s) \int_{W} \int_{V} f(v) d\left(p_{s} * p_{w}^{v}\right)(v) d m(w) .
$$

Since $(h f) *(h g)=h(f * g)$, we conclude that

$$
0=\int_{W} \int_{V}[h(v) h(w)-h(s)] f(v) d\left(p_{s} * p_{w}^{\vee}\right)(v) d m(w),
$$

contrary to the estimate

$$
\begin{aligned}
& \operatorname{Re} \int_{W} \int_{V}[h(v) h(w)-h(s)] \bar{\beta} f(v) d\left(p_{s} * p_{w}^{v}\right)(v) d m(w) \\
& \quad \geqq \eta \int_{W} \int_{V} f(v) d\left(p_{s} * p_{w}^{\vee}\right)(v) d m(w)=\eta \int_{W} f(s * \check{w}) d m(w)>0 ;
\end{aligned}
$$

see (1).

Thus $h(s)=h(x) h(y)$ for $s \in \operatorname{supp}\left(p_{x} * p_{y}\right)$ and hence $h(x * y)=$ $h(x) h(y)$. Since $h(x * e)=h(x) h(e)$ and $h$ is nonzero somewhere, we conclude that $h(e)=1$. Since $h(x) h(\breve{x})=h(e)=1$ and $h$ is bounded above, $|h|$ is bounded away from zero. Hence $|h|=1$ by $[9,4.2 .3]$ and so $h$ is in $Z\left(K^{\wedge}\right)$.

Corollary 3.7. Suppose that $\gamma \in Z\left(K^{\wedge}\right)$ and that $E$ is a spectral set (respectively, Calderón set or strong Ditkin set) in $K^{\wedge}$ for $A\left(K^{\wedge}\right)$. Then $\gamma E$ has the same property.

To check this corollary, use Lemma 2.2: $\left(J_{\gamma}(f)\right)^{\wedge}=(\hat{f})_{\bar{r}}$. The next theorem is immediate from Theorem 3.3 and Corollary 3.7.

THEOREM 3.8. Points in the center $Z\left(K^{\wedge}\right)$ of $K^{\wedge}$ are strong Ditkin sets for the algebra $A\left(K^{\wedge}\right)$. 
COROLLARY 3.9. Countable closed subsets of $Z\left(K^{\wedge}\right)$ are Calderón sets for $A\left(K^{\wedge}\right)$. See $[5,39.39 . c]$.

Theorem 3.8 is best possible in the sense that the points in $K^{\wedge} \backslash Z\left(K^{\wedge}\right)$ need not even be spectral sets; see (4.5), (4.7) and (4.8). Note, however, that Ditkin's condition holds at $\infty$ by Theorem 2.8.

A careful reading of (39.24), (39.39) and (39.42) in [5] shows that the following general result holds.

THEOREM 3.10. Let $\mathfrak{A}$ be a regular Banach algebra in $C_{0}(X)$, where $X$ is the structure space of $\mathfrak{A}$. Suppose that $\mathfrak{A}$ satisfies Ditkin's condition at $\infty$, and let $X_{0}$ denote the set of $x$ in $X$ at which $\mathfrak{A}$ satisfies Ditkin's condition.

(i) If $E$ is a closed subset of $X$ such that $\partial E \cong X_{0}$ and such that $\partial E$ contains no nonvoid perfect sets, then $E$ is a Calderón set for $\mathfrak{A}$.

(ii) If $E$ is a closed nonspectral subset of $X_{0}$, then there exists a continuum of closed ideals in $\mathfrak{A}$ with zero-set $E$.

CoROLlaRY 3.11. If $E$ is a closed subset of $K^{\wedge}$ such that $\partial E \subseteq$ $Z\left(K^{\wedge}\right)$ and $\partial E$ contains no nonvoid perfect sets, then $E$ is a Calderón set for $A\left(K^{\wedge}\right)$.

COROLLARY 3.12. Suppose that $K^{\wedge}$ is discrete at points of $K^{\wedge} \backslash Z\left(K^{\wedge}\right)$. If $E$ is closed in $K^{\wedge}$ and $E \cap Z\left(K^{\wedge}\right)$ contains no nonvoid perfect sets, then $E$ is a Calderón set. In particular, if $K^{\wedge} \backslash Z\left(K^{\wedge}\right)$ is discrete and $Z\left(K^{\wedge}\right)$ is countable, then every closed subset of $K^{\wedge}$ is a Calderón set.

See Example 4.6.

CoROLlaRY 3.13. If $E$ is a closed nonspectral set in $Z\left(K^{\wedge}\right)$, then there exists a continuum of closed ideals in $A\left(K^{\wedge}\right)$ with zero-set $E$.

Corollary 3.13 can fail if $E$ is a closed subset of $K^{\wedge}$; see (4.5).

4. Examples. In this section we give examples to show that points in $K^{\wedge}$ need not be spectral sets. We begin with a general discussion.

Let $A$ be a Banach algebra of continuous functions on a locally compact Hausdorff space $X$ such that $X$ can be considered as a subset of the structure space of $A$. The zero-set $Z(I)$ for a closed ideal $I$ in $A$ is $\{x \in X: f(x)=0$ for all $f \in I\}$. A closed subset $E$ of $X$ will 
be called a spectral set if it is not the zero-set of two distinct closed ideals in $A$. For a subset $S$ of the conjugate space $A^{*}$ of $A$, we write $I(S)$ for the set $\{f \in A: \varphi(f)=0$ for all $\phi \in S\}$. The set $I(S)$ need not be an ideal in $A$, but it will be under certain conditions on $S$.

Derivations 4.1. A set $D=\left\{d_{0}, d_{1}, \cdots, d_{n}\right\}$ in $A^{*}$ is often called a system of derivations if Leibniz rule

$$
d_{k}(f g)=\sum_{j=0}^{k}\left(\begin{array}{l}
k \\
j
\end{array}\right) d_{j}(f) d_{k-j}(g)
$$

holds for $f, g \in A, 0 \leqq k \leqq n$. Note that for $k=0$ this requirement simply asserts that $d_{0}$ is a multiplicative linear functional on $A$. If $d_{0}$ is point evaluation at $x_{0}$ in $X$, we call $D$ a system of derivations at $x_{0}$, and we call $d_{1}$ a point derivation at $x_{0}$.

It is easy to show that $D$ is a system of derivations in $A^{*}$, then $I(D)$ is a closed ideal in $A$. In particular, if $d_{1}$ is a point derivation at $x_{0}$ in $X$ and if $d_{0}$ denotes the evaluation map at $x_{0}$, then $I\left(\left\{d_{0}, d_{1}\right\}\right)$ is a closed ideal in $A$. If its zero-set is $\left\{x_{0}\right\}$, then $\left\{x_{0}\right\}$ is not a spectral set. For a partial converse, see Atzmon [1, 4.5].

In order to characterize spectral sets in $X$, we introduce a generalization of a "set of multiplicative linear functionals."

Collectionwise multiplicative sets 4.2. A subset $S$ of $A^{*}$ is called collectionwise multiplicative if, for $\varphi \in S$ and $f, g \in A$, there exist $\psi_{1}$ and $\psi_{2}$ in $S$ (depending on $\varphi, f$, and $g$ ) such that $\varphi(f g)=\psi_{1}(f) \psi_{2}(g)$ [2]. For a collectionwise multiplicative set $S$ in $A^{*}, I(S)$ is a closed ideal in $A$. In fact, it can be shown that a closed subset $S$ of $X$ is spectral if and only if for every collectionwise multiplicative set $T$ in $A^{*}$ with $Z(I(T))=S$ we have $I(T)=I(S)$.

There are various ways to obtain collectionwise multiplicative sets. If $D=\left\{d_{0}, d_{1}, \cdots, d_{n}\right\}$ is a system of derivations, then the absolutely convex hull of $\left\{2^{n} d_{0}, 2^{n-1} d_{1}, \cdots, 2 d_{n-1}, d_{n}\right\}$ is collectionwise multiplicative. Clearly the union of collectionwise multiplicative sets is collectionwise multiplicative, and so is its absolutely convex closed hull. More generally, suppose that $S$ is a collectionwise multiplicative set, that $a \geqq 1$, and that $T \cong A^{*}$ has the property that if $\varphi \in T$ and $f, g \in A$, then there exist $\psi \in S$ and complex numbers $\alpha, \beta, \gamma, \delta$ such that $\varphi(f g)=\alpha \varphi(f) \varphi(g)+\beta \varphi(f) \psi(g)+\gamma \psi(f) \varphi(g)+\delta \psi(f) \psi(g)$ and $|\alpha|+|\beta|+|\gamma|+|\delta| \leqq \alpha$. Then $a\langle T \cup S\rangle$ is collectionwise multiplicative where $\langle T \cup S\rangle$ is the absolutely convex closed hull of $T \cup S$. 
Point derivations in $M(K)$ 4.3. Now we give a general method to obtain point derivations for the algebra $M(K)$ which can be considered as a Banach algebra of bounded continuous functions on $\mathscr{X}_{b}(K)$ and $M(K)$ can be regarded as a Banach algebra of functions on $K^{\wedge}$ by $[6,6.3,7.3 \mathrm{E}]$.

Let $K$ be a commutative hypergroup such that $K^{\wedge}$ is a hypergroup. Let $\left\{\chi_{\alpha}\right\}$ be a net in $\mathscr{Z}_{b}(K),\left\{a_{\alpha}\right\}$ a net of complex numbers, $f$ a nonzero bounded continuous function on $K$, and $\chi \in \mathscr{Z}_{b}(K)$ such that $a_{\alpha}\left(\chi_{\alpha}-\chi\right) \rightarrow f$ and $\chi_{\alpha} \rightarrow \chi$ uniformly on compact subsets of $K$. Define $\Phi_{f}(\mu)=\int_{K} f d \mu$ for $\mu \in M(K)$, so that $\mathscr{X}_{b}(K)$ is embedded in the structure space of $M(K)$ via the mapping $\chi \rightarrow \Phi_{\chi}$. (Note that our $\Phi_{\chi}$ is the same as Jewett's $F_{\bar{\chi}}[6,6.3]$.) For $f$ described above, $\Phi_{f}$ is a point derivation for $M(K)$ at $\chi \in \mathscr{Z}_{b}(K)$.

We check that $\Phi_{f}$ is a point derivation at $\chi$, noting first that $\Phi_{f}$ is clearly a nonzero continuous linear functional on $M(K)$. If $x, y \in K$, then $a_{\alpha}\left(\chi_{\alpha}-\chi\right) \rightarrow f$ uniformly on the (compact) support of $p_{x} * p_{y}$ and so

$$
\begin{aligned}
f(x * y) & =\int_{K} f d\left(p_{x} * p_{y}\right)=\lim _{\alpha} a_{\alpha} \int_{K}\left[\chi_{\alpha}-\chi\right] d\left(p_{x} * p_{y}\right) \\
& =\lim _{\alpha} a_{\alpha}\left[\chi_{\alpha}(x) \chi_{\alpha}(y)-\chi(x) \chi(y)\right] \\
& =\lim _{\alpha}\left\{a_{\alpha}\left[\chi_{\alpha}(x)-\chi(x)\right] \chi_{\alpha}(y)+\chi(x) a_{\alpha}\left[\chi_{\alpha}(y)-\chi(y)\right]\right\} \\
& =f(x) \chi(y)+\chi(x) f(y) .
\end{aligned}
$$

Now for $\mu, \nu \in M(K)$, we have

$$
\begin{aligned}
\Phi_{f}(\mu * \nu) & =\int_{K} f d(\mu * \nu)=\int_{K} \int_{K} f(x * y) d \mu(x) d \nu(y) \\
& =\int_{K} \int_{K} f(x) \chi(y) d \mu(x) d \nu(y)+\int_{K} \int_{K} \chi(x) f(y) d \mu(x) d \nu(y) \\
& =\Phi_{f}(\mu) \Phi_{\chi}(\nu)+\Phi_{\chi}(\mu) \Phi_{f}(\nu),
\end{aligned}
$$

i.e., $\Phi_{f}$ is a point derivation for $M(K)$ at $\chi$. Note that $\Phi_{f}$ can, of course, also be regarded as a point derivation for $L^{1}(K)$ at $\chi$.

For a subset $\mathscr{F}$ of the space $C_{b}(K)$ of bounded continuous functions on $K$, we write $I(\mathscr{F})$ for the following closed subspace of $L^{1}(K):\left\{g \in L^{1}(K): \Phi_{f}(g)=0\right.$ for all $\left.f \in \mathscr{F}\right\}$. The following simple observation will help us determine when our ideals are distinct.

LEMMA 4.4. If $\mathscr{F}_{1}$ and $\mathscr{F}_{2}$ are distinct finite subsets of a linearly independent subset $\mathscr{F}$ of $C_{b}(K)$, then $I\left(\mathscr{F}_{1}\right) \neq I\left(\mathscr{F}_{2}\right)$.

Proof. $\left\{\Phi_{f}: f \in \mathscr{F}\right\}$ is a linearly independent set of linear func- 
tionals on $L^{1}(K)$. The lemma follows from an elementary fact concerning linear functionals [5, E. 12].

EXAMPLE 4.5. Let $G=\boldsymbol{R}^{n}$ and let $B$ be the group of rotations in $G$. The study of rotation-invariant functions and measures on $\boldsymbol{R}^{n}$ can be viewed as a study of functions and measures on the hypergroup $K=G_{B}$; see (1.2). As a set, $K$ is identified with $R^{+}=$ $[0, \infty)$; see the discussion and references in $[10, \S 3]$. The hypergroup $K^{\wedge}$ is isomorphic with $K$ and so $L^{1}(K)$ and $A(K)$ are isometrically isomorphic. The center of $K$ is $\{0\}$ and this is a Calderón set by Theorem 3.8. The remaining points of $K$ are nonspectral sets if $n \geqq 3$. This follows from the work of Reiter [7] which shows the connection between radial functions on $\boldsymbol{R}^{n}$ and L. Schwartz's original example of a nonsynthesis set. Varopoulos [14] has determined completely the closed ideals of $A(K)$ with zero-set $\{\rho\}$ in $(0, \infty)$.

Let us briefly cast these known results of Reiter and Varopoulos in terms of the notations in 4.1 and 4.2. Let $A^{\infty}(K)$ be the space of infinitely differentiable functions in $A(K)$. Fix $\rho$ in $(0, \infty)$ and let $\delta^{0}$ denote the point evaluation functional on $A(K): \delta^{0}(f)=f(\rho)$ for $f \in A(K)$. Similarly, let $\delta^{k}$ denote the $k$ th-derivative evaluated at $\rho: \delta^{k}(f)=f^{(k)}(\rho)$ for $f \in A^{\infty}(K)$. For $n \geqq 3, \delta^{1}$ is continuous in the topology of $A(K)[8, \mathrm{Ch} .2,6.3(4)]$ and can be defined on all of $A(K)$. Accordingly $\delta^{1}$ is a point derivation at $\rho$ and $\{\rho\}$ is not a spectral set as discussed in (4.1). Varopoulos [14, p. 384] shows that $\delta^{k}$ is continuous on $A(K)$ if and only if $k \leqq K_{n}$ where $K_{n}$ is the greatest integer less than or equal to $(1 / 2)(n-1)$. For $k \leqq K_{n}$, the set $D_{k}=$ $\left\{\delta^{0}, \delta^{1}, \cdots, \delta^{k}\right\}$ is a system of derivations at $\rho$ and $I\left(D_{k}\right)$ is a closed ideal in $A(K)$. These ideals are distinct and they all have zero-set $\{\rho\}$. Varopoulos shows that these are the only closed ideals with zero-set $\{\rho\}$. In particular, all points in $K$ are spectral sets if $n=2$.

EXAMPLE 4.6. Let $G$ be the group $\Delta_{p}$ of $p$-adic integers and let $B$ denote the group of units in $G$ acting on $G$. Then $G_{B}$ is a compact hypergroup identified with the one-point compactification $Z_{+}^{*}$ of $Z_{+}=$ $\{0,1,2, \cdots\}$. The hypergroup $K=G_{B}^{\hat{B}}$ is identified with $Z_{+}$and $G_{B}=K^{\wedge}$; see [4] for more details. Since the center of $G_{B}$ is $\{\infty\}$, Corollary 3.12 shows that every closed subset of $G_{B}$ is a Calderón set. For a class of hypergroups that includes $G_{B}$, Dunkl and Ramirez $[4,10.6]$ prove that every subset is a spectral set.

EXAMPLE 4.7. As in $[6,15.4]$, let $F$ be the hypergroup of conjugacy classes of the compact group $S U(2)$. Then $K=F^{\wedge}$ is a commutative discrete hypergroup and Proposition 1.4 shows that $K^{\wedge}$ is 
a hypergroup isomorphic with $F$ and that $\mathscr{Z}_{b}(K)=K^{\wedge}$. We may take $\{0,1,2, \cdots\}$ as a model for $K$ and $[0, \pi]$ as a model for $K^{\wedge}=F$. Specifically, $K^{\wedge}=\left\{\chi_{\theta}: \theta \in[0, \pi]\right\}$ where $\chi_{\theta}(n)=\sin (n+1) \theta /[(n+1) \sin \theta]$ for $\theta \in(0, \pi), \chi_{0}(n)=1$ and $\chi_{\pi}(n)=(-1)^{n}$ for $n=0,1,2, \cdots$. Since $Z\left(K^{\wedge}\right)=\left\{\chi_{0}, \chi_{\pi}\right\}$, the sets $\left\{\chi_{0}\right\},\left\{\chi_{\pi}\right\}$ are strong Ditkin sets.

We now show that for $\theta \in(0, \pi),\left\{\chi_{\theta}\right\}$ is not a spectral set. Let

$$
g_{\theta}(n-1)=\frac{d}{d \theta}\left(\chi_{\theta}(n-1)\right)=\frac{n \cos n \theta \sin \theta-\sin n \theta \cos \theta}{n \sin ^{2} \theta}
$$

for $n \geqq 1$. Then $g_{\theta}$ is a bounded (continuous) function on $K$ and hence $\Phi_{g_{\theta}}$ is a continuous linear functional on $M(K)$. Since $\chi_{\theta+\delta} \rightarrow \chi_{\theta}$ and $\delta^{-1}\left[\chi_{\theta+\delta}-\chi_{\theta}\right] \rightarrow g_{\theta}$ uniformly on compact subsets of $K$ as $\delta \rightarrow 0$, $\Phi_{g_{\theta}}$ is a point derivation at $\chi_{\theta}$ by (4.3). Since $\left\{\chi_{\theta}, g_{\theta}\right\}$ is linearly independent, the closed ideals $I\left(\left\{\chi_{\theta}\right\}\right)$ and $I\left(\left\{\chi_{\theta}, g_{\theta}\right\}\right)$ are distinct by (4.4). Each of these ideals has zero-set $\left\{\chi_{\theta}\right\}$ and so $\left\{\chi_{\theta}\right\}$ is not a spectral set.

NAIMARK's EXAMPLE 4.8. We assume familiarity with Jewett's treatment of Naimark's example $K=[0, \infty)[6,9.5]$. If $\operatorname{supp}(\pi)$ denotes the support of the Plancherel measure on $K^{\wedge}$, then $\operatorname{supp}(\pi) \subsetneq K^{\wedge} \subsetneq \mathscr{X}_{b}(K)$ and $1 \notin \operatorname{supp}(\pi)$. In particular, $K^{\wedge}$ is not a hypergroup. We will show that many points of $\mathscr{X}_{b}(K)$ are not spectral sets for $L^{1}(K)$ and, in fact, we will show that they are the zero-sets of infinitely many closed ideals.

Fix complex numbers $a, b$ with $a=b^{2}, a \neq 0$, and $0 \leqq \operatorname{Im}(b)<1$. For $n=0,1,2, \cdots$, let

$$
f_{2 n}(x)=x^{2 n} \sin b x / \sinh x \text { and } f_{2 n+1}(x)=x^{2 n+1} \cos b x / \sinh x .
$$

Note that $b^{-1} f_{0}=\chi_{a}$, an element of $\mathscr{X}_{b}(K)$. Each $f_{m}$ is a bounded continuous function on $K$. So each $\Phi_{f_{m}}$ is a continuous linear functional on $M(K)$. Straightforward calculations show that

$$
b f_{m}(x * y)=\sum_{j=0}^{m}\left(\begin{array}{c}
m \\
j
\end{array}\right)(-1)^{j(m+1)} f_{j}(x) f_{m-j}(y)+m(-1)^{m} f_{m-1}(x * y)
$$

for $x, y \in K$. It follows that

$$
b \Phi_{f_{m}}(\mu * \nu)=\sum_{j=0}^{m}\left(\begin{array}{c}
m \\
j
\end{array}\right)(-1)^{j(m+1)} \Phi_{f_{j}}(\mu) \Phi_{f_{m-j}}(\nu)+m(-1)^{m} \Phi_{f_{m-1}}(\mu * \nu)
$$

for $\mu, \nu \in M(K)$. Hence for any $m \geqq 0, I_{m}=I\left(\left\{f_{0}, f_{1}, \cdots, f_{m}\right\}\right)$ is a closed ideal in $L^{1}(K)$. Since $\left\{f_{0}, f_{1}, \cdots\right\}$ is a linearly independent set of functions on $K$, Lemma 4.4 shows that the ideals $I_{0}, I_{1}, \cdots$ are 
all distinct. Moreover, each of these ideals has zero-set $\left\{\chi_{a}\right\}$ in $\mathscr{Z}_{b}(K)$.

Similar computations show that $\left\{\chi_{0}\right\}$ is the zero-set of infinitely many closed ideals in $L^{1}(K)$. The auxiliary functions $f_{n}$ in this case are defined by $f_{n}(x)=x^{2 n+1} / \sinh x$. Note that $f_{0}=\chi_{0}$.

We do not know the status of the identity $\chi_{-1}$ or the other points of $\mathscr{Z}_{b}(K)$ not covered by the preceding discussion. However, we observe that no point of $\operatorname{supp}(\pi)=\left\{\chi_{t}: t \in[0, \infty)\right\}$ is a spectra] set.

The first author should like to thank the University of Oregon for giving her a visiting appointment and providing support and facilities during the academic session 1976-77.

\section{REFERENCES}

1. A. Atzmon, Spectral synthesis in regular Banach algebras, Israel J. Math., 8 (1970), 197-212.

2. A. K. Chilana, Topological algebras with a given dual, Proc. Amer. Math. Soc., 42 (1974), 192-197.

3. C. F. Dunkl, The measure algebra of a locally compact hypergroup, Trans. Amer. Math. Soc., 179 (1973), 331-348.

4. C. F. Dunkl and D. E. Ramirez, $A$ family of countable compact $P_{*}$-hypergroups, Trans. Amer. Math. Soc., 202 (1975), 339-356.

5. E. Hewitt and K. A. Ross, Abstract Harmonic Analysis. I and II, Springer-Verlag, 1963 and 1970.

6. R. I. Jewett, Spaces with an abstract convolution of measures, Advances in Math., 18 (1975), 1-101.

7. H. Reiter, Contributions to harmonic analysis. IV, Math. Annalen 135 (1958), 467-476.

8. Classical harmonic analysis and locally compact groups, Oxford Mathematical Mongraphs, 1968.

9. M. A. Rieffel, A characterization of commutative group algebras and measure algebras, Trans. Amer. Math. Soc., 116 (1965), 32-65.

10. K. A. Ross, Hypergroups and centers of measure algebras, Ist. Naz. Alta Mat. (Symposia Math.), volume XXII, (1977), 189-203.

11. - Centers of hypergroups, Trans. Amer. Math. Soc., to appear.

12. R. Spector, Apercu de la theorie des hypergroupes, Lecture Notes in Mathematics \#497. (Analyse Harmonique sur les Groupes de Lie, Sem. Nancy-Strasburg 1973-1975), Springer-Verlag.

13. - Measures invariantes sur les hypergroupes, to appear.

14. N. Th. Varopoulos, Spectral synthesis on spheres, Proc. Camb. Phil. Soc., 62 (1966), 379-387.

15. E. Kaniuth and D. Steiner, On complete regularity of group algebras, Math. Ann., 204 (1973), 305-329.

16. R. D. Mosak, The $L^{1}$ - and $C^{*}$-algebras of $[F I A]_{B}^{-}$groups, and their representations, Trans. Amer. Math. Soc., 163 (1972), 277-310.

Received August 8, 1977.

UNIVERSITY OF DELHI

DELHI 110007, INDIA

AND

UNIVERSITY OF OREGON

EUGENE, OR 97403 


\section{PACIFIC JOURNAL OF MATHEMATICS}

\section{EDITORS}

RICHARD ARENS (Managing Editor)

University of California

Los Angeles, CA 90024

Charles W. Curtis

University of Oregon

Eugene, OR 97403

C. C. MOORE

University of California

Berkeley, CA 94720

\section{J. DugundJI}

Department of Mathematics

University of Southern California

Los Angeles, CA 90007

R. FinN and J. Milgram

Stanford University

Stanford, CA 94305

\section{ASSOCIATE EDITORS}
E. F. BECKENBACH
B. H. NeumanN
F. WOLF
K. YosHIDA

\section{SUPPORTING INSTITUTIONS}

\author{
UNIVERSITY OF BRITISH COLUMBIA \\ CALIFORNIA INSTITUTE OF TECHNOLOGY \\ UNIVERSITY OF CALIFORNIA \\ MONTANA STATE UNIVERSITY \\ UNIVERSITY OF NEVADA, RENO \\ NEW MEXICO STATE UNIVERSITY \\ OREGON STATE UNIVERSITY \\ UNIVERSITY OF OREGON
}

\author{
UNIVERSITY OF SOUTHERN CALIFORNIA \\ STANFORD UNIVERSITY \\ UNIVERSITY OF HAWAII \\ UNIVERSITY OF TOKYO \\ UNIVERSITY OF UTAH \\ WASHINGTON STATE UNIVERSITY \\ UNIVERSITY OF WASHINGTON
}

The Supporting Institutions listed above contribute to the cost of publication of this Journal, but they are not owners or publishers and have no responsibility for its content or policies.

Mathematical papers intended for publication in the Pacific Journal of Mathematics should be in typed form or offset-reproduced, (not dittoed), double spaced with large margins. Please do not use built up fractions in the text of the manuscript. However, you may use them in the displayed equations. Underline Greek letters in red, German in green, and script in blue. The first paragraph or two must be capable of being used separately as a synopsis of the entire paper. Items of the bibliography should not be cited there unless absolutely necessary, in which case they must be identified by author and journal, rather than by item number. Manuscripts, in triplicate, may be sent to any one of the editors. Please classify according to the scheme of Math. Reviews, Index to Vol. 39. All other communications should be addressed to the managing editor, or Elaine Barth, University of California, Los Angeles, California, 90024.

50 reprints to each author are provided free for each article, only if page charges have been substantially paid. Additional copies may be obtained at cost in multiples of 50 .

The Pacific Journal of Mathematics is issued monthly as of January 1966. Regular subscription rate: $\$ 72.00$ a year (6 Vols., 12 issues). Special rate: $\$ 36.00$ a year to individual members of supporting institutions.

Subscriptions, orders for numbers issued in the last three calendar years, and changes of address should be sent to Pacific Journal of Mathematics, 103 Highland Boulevard, Berkeley, California, 94708. Older back numbers obtainable from Kraus Periodicals Co., Route 100, Millwood, NY 10546.

PUBLISHED BY PACIFIC JOURNAL OF MATHEMATICS, A NON-PROFIT CORPORATION

Printed at Kokusai Bunken Insatsusha (International Academic Printing Co., Ltd.). 8-8, 3-chome, Takadanobaba, Shinjuku-ku, Tokyo 160, Japan.

Copyright (C) 1978 by Pacific Journal of Mathematics Manufactured and first issued in Japan 


\section{Pacific Journal of Mathematics}

\section{Vol. 76, No. $2 \quad$ December, 1978}

Stephanie Brewster Brewer Taylor Alexander, Local and global convexity in complete Riemannian manifolds ...........................

Claudi Alsina i Català, On countable products and algebraic convexifications of probabilistic metric spaces ...............................

Joel David Berman and George Grätzer, Uniform representations of

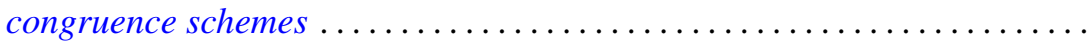

Ajit Kaur Chilana and Kenneth Allen Ross, Spectral synthesis in

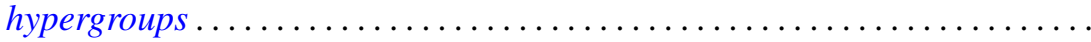

David Mordecai Cohen and Howard Leonard Resnikoff, Hermitian quadratic forms and Hermitian modular forms . .........................

Frank Rimi DeMeyer, Metabelian groups with an irreducible projective

representation of large degree .............................

Robert Ellis, The Furstenberg structure theorem .....................

Heinz W. Engl, Random fixed point theorems for multivalued mappings .......

William Andrew Ettling, On arc length sharpenings ..................

Kent Ralph Fuller and Joel K. Haack, Rings with quivers that are trees........

Kenneth R. Goodearl, Centers of regular self-injective rings ...............

John Gregory, Numerical algorithms for oscillation vectors of second order

differential equations including the Euler-Lagrange equation for

symmetric tridiagonal matrices.

Branko Grünbaum and Geoffrey Shephard, Isotoxal tilings

Myron Stanley Henry and Kenneth Leroy Wiggins, Applications of

approximation theory to differential equations with deviating

arguments

Mark Jungerman, The non-orientable genus of the n-cube .

Robert Richard Kallman, Only trivial Borel measures on $S_{\infty}$ are

quasi-invariant under automorphisms ................

Joyce Longman and Michael Rich, Scalar dependent algebras in the

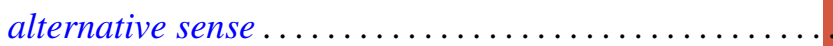

Richard A. Mollin, The Schur group of a field of characteristic zero ........ 471

David Pokrass, Some radical properties of rings with $(a, b, c)=(c, a, b) \ldots 479$

Margaret Shay and Paul Ruel Young, Characterizing the orders changed by

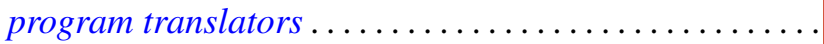

Jerrold Norman Siegel, On the structure of $B_{\infty}(F), F$ a stable space...

Surjeet Singh, (hnp)-rings over which every module admits a basic

submodule...

A. K. Snyder, Universal interpolating sets and the Nevanlinna-Pick property in

Banach spaces of functions...

Jeffrey D. Vaaler, On the metric theory of Diophantine approximation ... 